\title{
STATISTICAL APPROXIMATION OF FOURIER TRANSFORM-IR SPECTROSCOPY DATA FOR ZINC WHITE PIGMENT FROM TWENTIETH-CENTURY RUSSIAN PAINTINGS
}

\author{
I. A. Balakhnina, ${ }^{a, b^{*}}$ N. N. Brandt, ${ }^{\text {a }}$ D. Valenti, ${ }^{b}$ \\ I. A. Grigorieva, ${ }^{\text {c }}$ B. Spagnolo, ${ }^{\text {b }}$ and A. Yu. Chikishev ${ }^{a}$
}

UDC 535.34:667.622.114.712

We present a statistical model for approximation of experimental Fourier transform-IR spectroscopy (FTIR) data for paint samples from paintings of different ages. The model utilizes random variations in some parameters (initial ageing rate, degree of change in ageing rate and time at which the change occurs). We determine the parameters characterizing variation in the paint composition and the storage conditions for the paintings. The numerical calculation is qualitatively consistent with the experimental data. In the proposed model, changes in the initial composition of the paint and the storage conditions make about the same contribution to the experimentally observed scatter in the data points.

Keywords: IR spectroscopy, dating, zinc white, simulation.

Introduction. Ageing of a paint layer is associated with various physical and chemical processes, in particular with polymerization and oxidation as the binders dry out, and also with chemical reactions when the binders react with the pigments, resulting in formation of new substances (compounds). Photoinduced changes in the binders and pigments, their chemical reactions during storage of the paintings (especially when the atmosphere is significantly polluted), as well as mechanical damage to the paint and varnish layers are also possible. Study of processes associated with ageing of paintings is important for modern applied science. The corresponding results allow us to solve a number of practical problems such as restoration, determination of authenticity, and dating.

Several experimental methods can be used to determine the composition of the paint layer in a painting. Optical spectroscopy is a useful tool in analysis of the molecular composition of samples (see, for example, [1, 2]). The position and relative intensities of the spectral bands can be used to determine structural changes in the analyte molecules. Fourier transform-IR (FTIR) microspectroscopy is often used as a highly sensitive method, making it possible to work with micronsized samples [3]. Databases for IR spectra of paints and binders are available for interpretation of the experimental results $[4,5]$. Ageing causes changes in the molecular composition of the paint layers. Measurements of the spectroscopic parameters (line intensities and positions) probably can be used to determine the age of a painting. In fact, monotonic changes in the spectral lines of the oil after drying out have been demonstrated in [6]. However, polymerization and oxidation processes associated with ageing of the paint layer depend considerably on the environment (temperature, humidity, and composition of the atmosphere) and the illumination conditions. Thus identical spectral changes can be observed for paintings of different ages while the measured parameters can be quite different for two paintings of the same age.

In order to solve this problem, after determining the time-dependent parameter for a set of paintings of different ages, we use statistical data processing methods. Then for an unknown painting, the parameter can be used to estimate the age. Such an approach requires knowing a specific mathematical model for ageing, making it possible to calculate an analytical "dating curve". Nevertheless, analysis of experimental data for a statistically significant number of paintings has shown that such an approach does not work due to the significant scatter in the experimental data and the lack of an adequate analytical model for ageing of paintings under unknown conditions [7]. Furthermore, the results of averaging have shown that the nature of the dependences under study are considerably nonlinear (threshold type) [7].

\footnotetext{
* To whom correspondence should be addressed.
}

${ }^{\mathrm{a}}$ Physics Department and International Laser Center, M. V. Lomonosov Moscow State University, 1 Leninskie Gory, Moscow, 119991, Russia; e-mail: balakhnina@physics.msu.ru; ${ }^{b}$ University of Palermo, Palermo, Italy; ${ }^{\mathrm{c}}$ State Hermitage Museum, St. Petersburg, Russia. Translated from Zhurnal Prikladnoi Spektroskopii, Vol. 84, No. 3, pp. 469-474, May-June, 2017. Original article submitted September 6, 2016. 


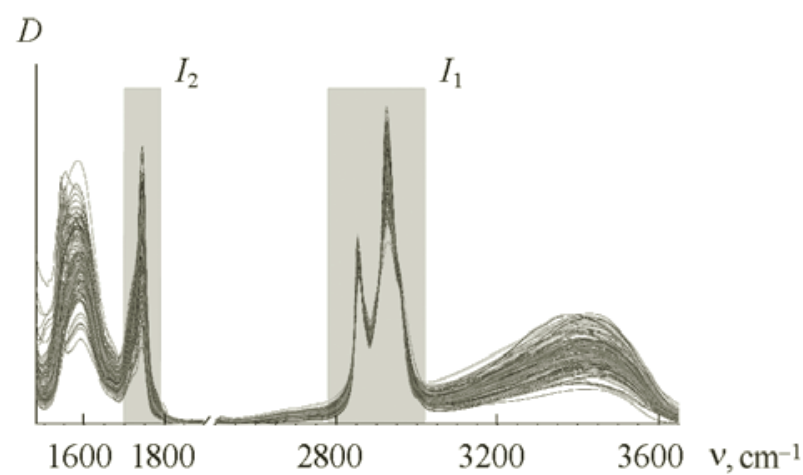

Fig. 1. FTIR spectra of 192 samples of zinc white after computer processing.

We start from the hypothesis that significant changes in the measured spectroscopic parameters (for example, the line intensities) with the age of the painting can be associated with variation in both the original compositions of the paints and also the storage conditions for the painting. We introduce a parameter characterizing the closeness between the model and the experiment which is not the mean-square deviation. The aim of this work was to develop a statistical (nonanalytical) model for approximation of previously obtained experimental data and to determine the parameters characterizing the variation in the paint composition and the storage conditions for the paintings.

The Experiment. We analyzed 493 zinc white paint samples, taken from 230 paintings by Russian artists (A. K. Savrasov, K. E. Makovsky, P. P. Konchalovsky, M. F. Larionov, V. V. Kandinsky, N. I. Altman, K. S. Malevich, B. M. Kustodiev, A. M. Rodchenko, R. R. Falk, and A. V. Lentulov). The paintings were provided by several Russian museums and also private collectors. The artist and the creation date are indicated on most of the paintings. There are art expert assessments for all the paintings.

Samples were taken under an optical microscope. The sample size was on the order of fractions of a millimeter. The samples were cleaned to remove foreign particulates. From the entire collection of samples, we selected $N=219$ in which we confirmed the presence of zinc white and the absence of lead white using X-ray spectral microanalysis. Note that $M=54$ different ages of the paintings corresponds to the 219 analyte samples (there are several samples for each age of a painting, except for four). For spectroscopic analysis, fragments of size 20-50 $\mu \mathrm{m}$ were mechanically removed from the interior of the sample without using organic solvents. In such fragments, there are practically no elements from the varnish and underlying layers. A roller knife was used to make thin layers from the fragments on $\mathrm{KBr}$ plates.

The measurements on the FTIR transmission spectra were done on a Hyperion 1000 microscope (Bruker) with a Tensor 37 spectrometer [8] in the $600-4000 \mathrm{~cm}^{-1}$ range, with resolution $4 \mathrm{~cm}^{-1}$. Lateral resolution $20 \mu \mathrm{m}$. The lines in the IR spectra were identified using the data in [6, 9-11].

Results and Discussion. For processing and comparing the spectra, we used an algorithm for approximating one spectrum by another spectrum with addition of a polynomial of a given degree, which allows us to do a comparative analysis of the spectra without resorting to elimination of the background signal [12]. Note that the lines for vibrations of zinccontaining molecular groups lie outside the indicated spectral range. All the observable lines are assigned to the oil binder, while the $1650 \mathrm{~cm}^{-1}$ line is assigned to the reaction products between the oil and the pigment. The spectra are normalized to the integrated intensity $I_{1}$ in the $2780-3020 \mathrm{~cm}^{-1}$ range (interval $I_{1}$ in Fig. 1). The spectral components in this range correspond to symmetric $\left(2850 \mathrm{~cm}^{-1}\right)$ and antisymmetric $\left(2930 \mathrm{~cm}^{-1}\right)$ stretching vibrations of the $\mathrm{CH}_{2}$ groups and symmetric $\left(2870 \mathrm{~cm}^{-1}\right)$ and antisymmetric $\left(2960 \mathrm{~cm}^{-1}\right)$ stretching vibrations of the $\mathrm{CH}_{3}$ groups [13]. We can see that the intensity $I_{1}$ does not change with the age of the painting [7].

The band at frequency $1740 \mathrm{~cm}^{-1}$ corresponds to vibrations of the carbonyl groups and has a shoulder at $1709 \mathrm{~cm}^{-1}$ (carboxyl groups and/or free carboxylates) [14]. The intensity of this band decreases during polymerization and oxidation of the oil [6]. Let us introduce the parameter $Y=I_{2} / I_{1}$, where $I_{2}$ is the integrated intensity in the range $1700-1790 \mathrm{~cm}^{-1}$. Let us consider the dependence of $Y$ on the age of the painting (Fig. 2a, points). Note the considerable scatter in the experimental data. Since the mean-square deviation of the $Y$ values obtained for different samples from the same painting is not greater than 0.03 , we can say that this scatter is mainly due to differences between the oxidation and/or polymerization processes in the oil paints on different canvases. As noted in [7], approximation of the presented data using any analytical function is not a valid procedure. However, we can propose a theoretical model including a number of random parameters. 

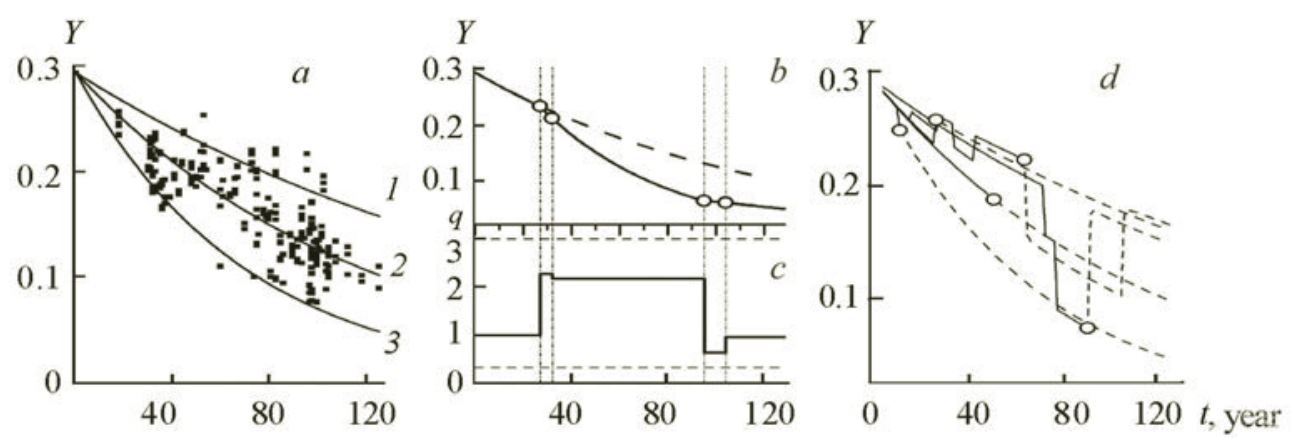

Fig. 2. The parameter $Y$ vs. the age of the painting: a) experimental results (points) and calculated ageing curves for $a=a_{0} / 1.7$ (1), $a_{0}(2)$, and $1.7 a_{0}(3)$; b) calculated ageing curve (dashed line) for constant storage conditions and for changes in ageing conditions (solid line); c) change in ageing rate; d) determination of points approximating the experimental data.

Since the values of $Y$ may differ for paintings of the same age by an amount considerably greater than the scatter in $Y$ for a single painting, it is logical to hypothesize that there exists some parameter characterizing the ageing rate for zinc white in each painting. This parameter can be determined by the presence in the paint of components accelerating its drying (dessicants), and/or it can vary over time in connection with a change in the storage conditions for the painting.

In order to study the dependence of $Y$ on the age $t$ of the painting, we constructed a model with the following conditions and assumptions:

1. At the time the picture was painted (when $t=0$ ), the value of $Y$ is maximum: $\max Y=Y(0) \equiv Y_{0}$. In order to determine the value of $Y_{0}$, we measured the IR spectrum of fresh paint (zinc white), dried on canvas for 10 days under normal conditions $\left(Y_{0}=0.293\right)$.

2. If the storage conditions (exposure conditions) for the painting do not change, then $Y_{0}(t)=Y_{0} e^{-a t}$, where $a$ is the ageing rate of the paint on the painting.

3. The entire ensemble of experimental points is approximated by the function $Y(t)=0.293 e^{-a_{0} t}$. Figure 2a shows the approximation curve obtained with $a_{0}=0.00836$ year $^{-1}$.

4. Since the initial ageing rates of the paint are different for different paintings, we introduce the maximum difference coefficient $C>1$ indicating the difference between the initial ageing rate and the average rate $a_{0}$. For simulation of the experimental data, we randomly choose the sign for the change in the rate relative to the average, and then we choose the ageing rate of the current painting from the intervals $\left[a_{0}, C a_{0}\right]$ or $\left[a_{0} / C, a_{0}\right]$ depending on the sign for the change. Here and below, to randomly choose the value of the parameter from some interval we use the standard rand function of the MatLab environment. Figure 2a shows two limiting exponentials with $a=a_{0} / 1.7$ and $a=1.7 a_{0}$.

5. Changes in the storage conditions for the painting can lead to either an increase or a decrease in the ageing rate. The number of such changes and the time when they occur are random parameters. Figure $2 b$ shows the ageing curves for a single painting for unchanged conditions and for some changes in the storage conditions at the indicated times. For unchanged conditions, the ageing rate $a_{0}$ is constant. In the second case, at the initial time the rate is equal to $a_{0}$, and then changes abruptly (Fig. 2c) at the times when the age of the painting is 27, 32, 95, and 104 years.

Let us determine the maximum number $k$ of changes in the ageing rate during storage of the painting. Let us choose the actual number of changes in rate for the given painting randomly in the interval from 0 to $k$. Then let us choose the sign for the change in the rate of relative to the average and the ageing rate of the current painting from the intervals $\left[a_{0}, q a_{0}\right]$ or $\left[a_{0} / q, a_{0}\right]$ depending on the sign of the deviation. For the case shown in Fig. 2, we used the parameters $k=6$ and $q=3$.

6. The experimental data are a set of $N=219$ points, each of which corresponds to a single painting. We used the following procedure to simulate such a data set: Construct a set of $N$ curves with individual parameters $C, k$, and $q$. Compare each curve with one painting. On the curve, select a point corresponding to the age of the painting. As a result, obtain a set of $N$ points. For example, in Fig. 2d, we show five simulated curves and the corresponding points.

7. The problem of comparing the simulated and experimental sets of data points on the $(Y, t)$ coordinate plane requires separate consideration. At first glance, in order to compare the results of the calculation $Y^{\text {calc }}$ for different parameters $C, k$, and $q$ and the experimental data $Y^{\exp }$, it is logical to calculate a quantity analogous to the mean-square deviation: 


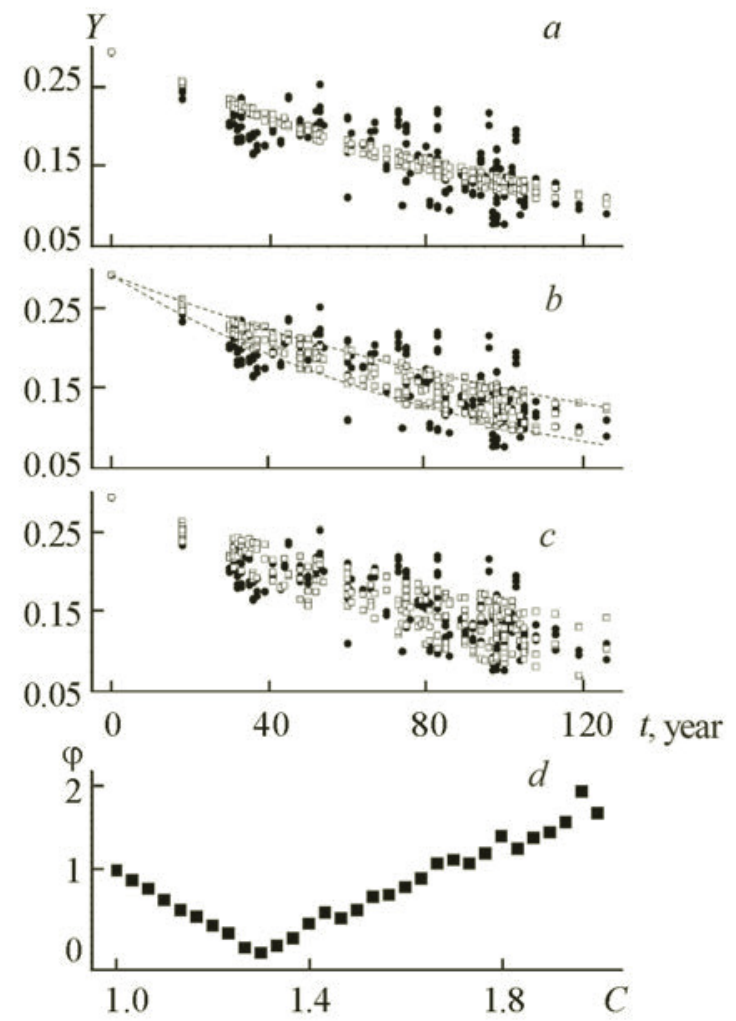

Fig. 3. The parameter $Y$ vs. age of the painting (experimental results $(\bullet)$ and approximation results ( $\square$ ) for $C=1.1$ (a), 1.3 (b), and 1.5 (c)) and the $\varphi(C)$ dependence for $k=0$ (d). Dashed lines: exponential curves with $a=a_{0} / 1.25$ and $1.25 a_{0}$.

$\sigma^{2}=(1 / N) \sum_{i=1}^{N=219}\left(Y_{i}^{\text {calc }}-Y_{i}^{\exp }\right)^{2}$, and tends toward its lowest possible value. However, calculated estimates show that $\sigma^{2}$ monotonically decreases as $C, k$, and $q$ increase, while the minimum value of $\sigma^{2}$ in this case corresponds to the curve $Y(t)=0.293 \exp \left(-a_{0} t\right)$.

In order to do a proper comparison, we suggest determining at least two parameters: the first characterizes the vertical shift of the data sets relative to each other, and the second characterizes the scatter in the data points, i.e., the area occupied by the data set on the coordinate plane. However, calculation of the first parameter is impractical, since in the suggested model the data points are automatically scattered relative to the curve $Y(t)=0.293 \exp \left(-a_{0} t\right)$. Consequently, the model already includes zero shift of the data sets relative to each other. Thus it is sufficient to just compare the areas of the data sets. For each age $t_{i}$ (except for four of the $M=54$ ages), several values of $Y$ are included in the experimental and consequently also in the simulated data sets, the maximum and minimum of which we denote as $Y_{i, \max }$ and $Y_{i, \min }$. Then the area of the data set $M=54$

is $S=\sum_{i=1}\left(Y_{i, \max }-Y_{i, \min }\right)$, and for comparison of the scatter of the points in the experimental and simulated data sets for different parameters $C, k$, and $q$, we can introduce the parameter $\varphi=\left|1-S_{\text {calc }} / S_{\text {exp }}\right|$.

Let us consider three variants for variation of the model parameters. In the first variant, the ageing rates $a$ for all the paintings are initially different, but do not change over the entire storage time for the painting $(k=0)$. Such an approximation is valid if the scatter in the experimental points is completely determined by variation in the original composition of the paint, and does not depend on the storage conditions for the painting. In Fig. 3a-c, we show the calculation results for $C=1.1,1.3$, and 1.5; in Fig. 3d, we show the $\varphi(C)$ dependence, having a minimum at $C=1.3$. Each point on the dependence is the result of averaging over 100 realizations of the numerical calculations. For convenience, the ageing process can be described by both the rate $a$ and the quantity $\tau=1 / a$, which we call the ageing time. For $C=1.3$, the ageing time interval $\left[1 /\left(C a_{0}\right), C / a_{0}\right]$ is $92-156$ years (in this case, $\tau_{0}=1 / a_{0}=120$ years). 


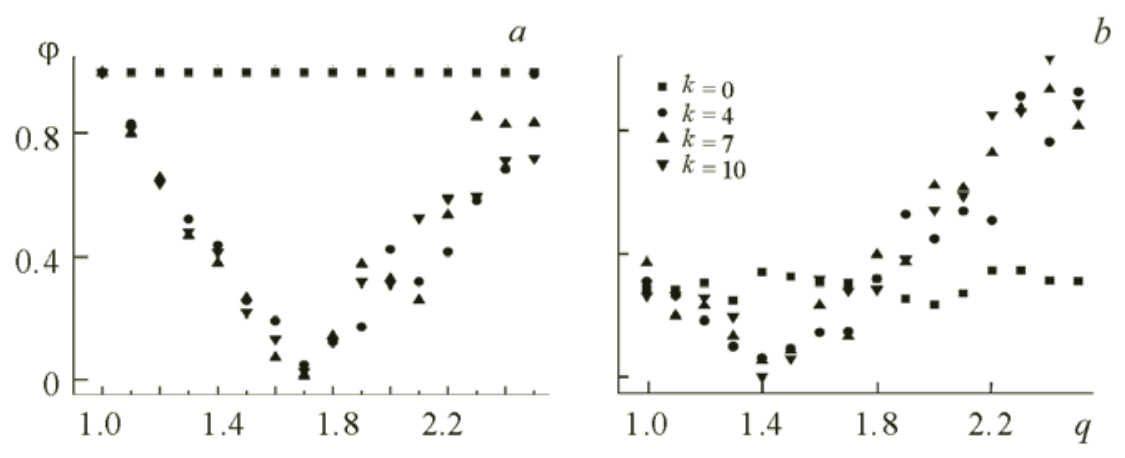

Fig. 4. The $\varphi(q)$ dependence in the case $C=1$ (a) and 1.2 (b).

In the second variant, the initial ageing rate for all the paintings is the same $(C=1)$, while changes in the rate occur during storage of the painting $(k \neq 0)$. Such a situation is realized in practice if the variation in the original composition of the paint makes a contribution to the observed scatter in the experimental points that is negligibly small compared with the contribution from changes in the storage conditions. In Fig. $4 \mathrm{a}$, we show the dependences $\varphi(q)$ for $k=0,4,7,10$. The minimum on the curves is achieved at $q \approx 1.7$, which corresponds to the interval [1/( $\left.\left.q a_{0}\right), q / a_{0}\right]$ of ageing times $71-204$ years.

In the third variant, the parameters $C$ and $q$ are varied simultaneously (for $k \neq 0$ ). Since for $C=1.3$ and $k=0$, the parameter $\varphi \approx 0$ (i.e., the model describes the experimental data well), we can hypothesize that introducing an additional independent parameter $q$ for $k \neq 0$ makes the results of the approximation worse for $C \geq 1.3$. The approximation result shows that as $C$ decreases, in order to achieve $\varphi \approx 0$, we need to decrease $q$ relative to the value of this parameter for $C=1$ (compare Fig. $4 \mathrm{a}$ and b). The smallest values $C_{\min }, q_{\min }$ for which $\varphi$ is minimum are equal to $1.25,1.35$. We see that in this case, the corresponding ageing time intervals $96-150$ and 89-161 years practically coincide. Thus within the proposed model, variations in the initial composition of the paint and changes in the storage conditions are equivalent effects, making a contribution to the scatter in the data points observed in the experiment (Fig. 2). Figure 3 b shows two curves corresponding to ageing times 96 and 150 years. 129 experimental points ( $60 \%$ of the samples) fall within the region bounded by these curves.

Conclusions. The ratio of the integrated intensities in the intervals $1700-1790$ and $2780-3020 \mathrm{~cm}^{-1}$ (the parameter $Y$ ) in the FTIR spectra of oil paints based on zinc white from paintings by Nineteenth-Century and Twentieth-Century Russian artists depends on the age of the paintings. However, the experimental data do not allow us to determine the analytical dependence of the parameter $Y$ on time due to the large scatter in its values even for paintings that were painted in the same year. Such scatter may be associated both with the difference between the original paint compositions and with different storage conditions for the painting, where the storage conditions can change several times for each painting. Within the model, we have approximated the experimental data, using random changes in some parameters of the model (the initial ageing rate, the degree of change in the ageing rate and the time at which the change occurs). In the proposed model, changes in the initial composition of the paint and the storage conditions make about the same contribution to the scatter in the data points observed in the experiment. Based on the calculations performed, we can say that the ageing time interval for the paintings from the considered set is $\approx 90-160$ years. In this case, the proposed model cannot be used for dating an individual painting with acceptable accuracy.

Acknowledgments. This work was done using equipment purchased with funds from the Development Program of Moscow State University, and with the financial support of the Russian Foundation for Basic Research as part of scientific projects 14-06-00259-a, 14-06-00244, and 17-06-00636.

\section{REFERENCES}

1. V. Desnica, K. Furic, B. Hochleitner, and M. Mantler, Spectrochem. Acta B, 58, 681-687 (2003).

2. R. J. H. Clark, Chem. Soc. Rev., 24, 187 (1995).

3. M. Derrick, D. Stulik, and J. Landry, Infrared Spectroscopy in Conservation Science, Getty Conservation Institute, Los Angeles (1999).

4. K. J. van den Berg, A. Burnstock, M. de Keijzer, J. Krueger, T. Learner, A. de Tagle, and G. Heydenreich (Eds.), Issues in Contemporary Oil Paint, Springer International, Switzerland (2014). 
5. S. Vahur, A. Teearu, P. Peets, L. Joosu, and I. Leito, Anal. Bioanal. Chem., 408, 3373-3379 (2016).

6. J. van der Weerd, A. van Loon, and J. J. Boon, Stud. Conserv., 50, No. 1, 3-22 (2005).

7. I. A. Balakhnina, N. N. Brandt, A. Y. Chikishev, Y. I. Grenberg, I. A. Grigorieva, I. F. Kadikova, and S. A. Pisareva, Appl. Spectrosc., 70, No. 7, 1150-1156 (2016); doi: 10.1177/0003702816652330.

8. Bruker Corporation, HYPERION Series FT-IR Microscopes; https://www.bruker.com/products/infrared-near-infraredand-raman-spectroscopy/ft-ir-microscopes-raman-microscopes/hyperion/overview.html [accessed December 4, 2015].

9. J. Mallegol, J.-L. Gardette, and J. Lemaire, J. Am. Oil Chem. Soc., 76, No. 8, 967-976 (1999).

10. J. Mallegol, J.-L. Gardette, and J. Lemaire, J. Am. Oil Chem. Soc., 77, No. 3, 249-255 (2000).

11. H. Kuhn, Zinc White. Artists Pigments: A Handbook of Their History and Characteristics, Cambridge University Press, Cambridge (1986).

12. N. N. Brandt, A. Yu. Chikishev, A. I. Chulichkov, P. A. Ignatiev, S. I. Lebedenko, and O. V. Voronina, Laser Phys., 14, No. 11, 1386-1392 (2004).

13. F. C. Izzo, K. J. van den Berg, H. van Keulen, B. Ferriani, and E. Zendri, J. Cultural Heritage, 15, No. 5, 557-563 (2014).

14. B. Smith, Infrared Spectral Interpretation: A Systematic Approach, CRC Press, London (1998). 